\title{
Temporal Stability of the Location of the Esophagus in Patients Undergoing a Repeat Left Atrial Ablation Procedure for Atrial Fibrillation or Flutter
}

\author{
ROBERT KENNEDY, M.D., ERIC GOOD, D.O., HAKAN ORAL, M.D., \\ ELIZABETH HUETHER, C.C.T, FRANK BOGUN, M.D., FRANK PELOSI, M.D., \\ FRED MORADY, M.D., and AMAN CHUGH, M.D.
}

From the Division of Cardiology, University of Michigan Health System, Ann Arbor, Michigan, USA

Esophageal Location. Background: The esophagus may be mobile during a left atrial (LA) ablation procedure for atrial fibrillation (AF).

Objective: The goal of the study was to determine whether the location of the esophagus is stable in patients undergoing a repeat $\mathrm{LA}$ ablation procedure.

Methods: Forty-two patients underwent repeat $L A$ ablation a mean of $7 \pm 2$ months after the initial procedure. Cinefluoroscopic images of the esophagus during a barium swallow were recorded and the course of the esophagus was tagged on the $3 \mathrm{D}$ map. The position of the esophagus at the index and repeat procedure were compared.

Results: At the index procedure, the esophagus was located near the left pulmonary veins (PVs) in 20 (48\%), right PVs in $13(31 \%)$, and at the mid LA in $9(21 \%)$ patients. During the repeat procedure, the esophagus was found to be near the left PVs in $22(52 \%)$, right PVs in $11(26 \%)$, and at the mid LA in 9 patients $(21 \%)$. In 35 of the 42 patients $(83 \%)$, there was no change in the esophageal location, and in the remaining seven patients $(17 \%)$, its position had shifted by $\geq 1 \mathrm{~cm}($ range $1.0-4.0 \mathrm{~cm})$.

Conclusions: In more than $80 \%$ of patients presenting for a repeat $L A$ ablation procedure, the esophagus is in the same position relative to the PVs as during the initial procedure. Therefore, if radiofrequency ablation at a particular location was limited by the position of the esophagus, safe ablation at that site is unlikely to be feasible during a repeat procedure. (J Cardiovasc Electrophysiol, Vol. 19, pp. 351-355, April 2008)

esophagus, atrial fibrillation, catheter ablation

A serious complication of radiofrequency catheter ablation of atrial fibrillation $(\mathrm{AF})$ is esophageal injury and the formation of an atrio-esophageal (AE) fistula. ${ }^{1,2}$ One strategy to reduce the risk of this complication is to avoid the posterior left atrium (LA) immediately over the esophagus. This approach requires real-time imaging of the esophagus during the ablation procedure. The location of the esophagus may limit successful ablation, ${ }^{3}$ resulting in arrhythmia recurrences and repeat procedures. As the esophagus may be a mobile structure, ${ }^{4}$ it is possible that it would be at a different location during the repeat procedure, thereby allowing a critical area to be safely ablated.

The purpose of this study was to compare the position of the esophagus relative to the PVs and posterior LA in patients who underwent two ablation procedures.

\section{Methods \\ Patient Characteristics \\ Forty-two consecutive patients underwent a repeat abla- tion procedure for either AF (16) or atypical atrial flutter (26)}

Address for correspondence: Aman Chugh, M.D., Cardiology, TC B1 D140, 1500 E. Medical Center Dr., Ann Arbor, MI 48109-0311. Fax: (734) 9367026; E-mail: achugh@umich.edu

Manuscript Received 15 September 2007; Revised manuscript received 11 October 2007; Accepted for publication 19 October 2007.

doi: 10.1111/j.1540-8167.2007.01051.x a mean of $7 \pm 2$ months after an initial AF ablation procedure. There were 29 men and 13 women. Their mean age, left ventricular ejection fraction, and left atrial (LA) diameter were $57 \pm 9$ years, $0.54 \pm 0.09,43 \pm 8 \mathrm{~mm}$, respectively. Prior to the first procedure, AF had been paroxysmal in 30 patients $(71 \%)$ and persistent in 12 patients $(21 \%)$. Twelve of the 42 patients (29\%) had structural heart disease, including hypertension with left ventricular hypertrophy in six, coronary artery disease in three, dilated cardiomyopathy in one, hypertrophic cardiomyopathy in one, and repair of an atrial septal defect in one.

\section{Ablation Procedure for $\mathrm{AF}$}

Patients with a history of persistent AF underwent transesophageal echocardiography prior to the procedure to rule out intracardiac thrombi. The ablation procedure for AF was guided by an electroanatomic mapping system (CARTO, Biosense-Webster, Diamond Bar, CA, USA). A 3D replica of the LA and tubular models of the PVs were created. The PV ostia were identified by a combination of venography, real-time impedance, ${ }^{5}$ characteristics of the local electrograms, and fluoroscopy. In 29 of 42 study patients (69\%), circumferential PV ablation was performed at the initial session. ${ }^{6}$ Briefly, circumferential lesions were deployed around the left- and right-sided PVs, along with linear ablation at the posterior wall and mitral isthmus. After AE fistula was described, ${ }^{1}$ the posterior line was either omitted or moved to the most cranial aspect of the roof. Six patients (14\%) underwent LA ablation that targeted complex, fractionated 
AP

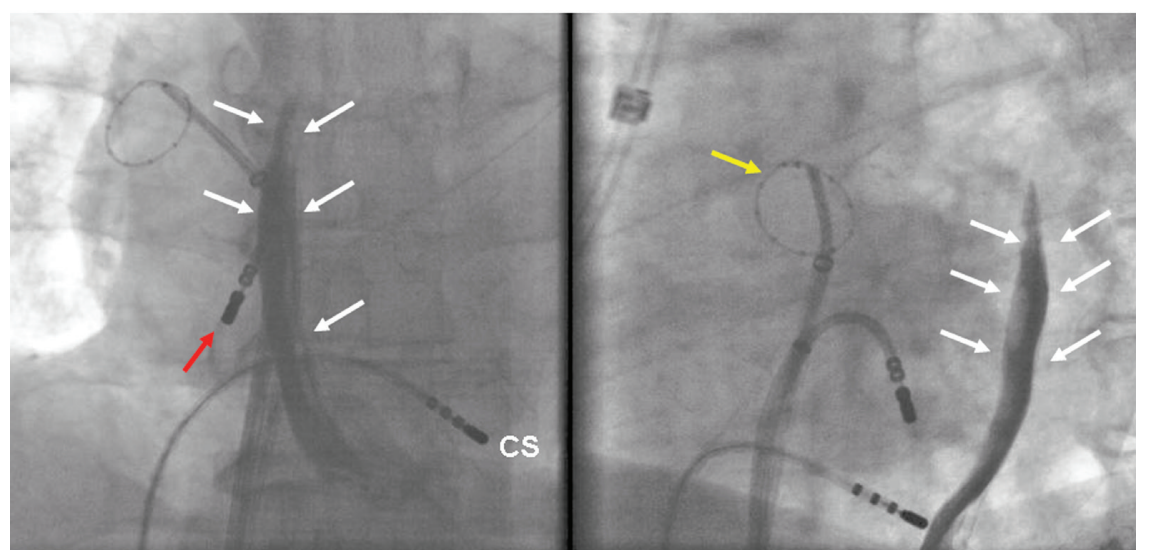

Figure 1. An esophagram obtained during a barium swallow in a patient undergoing an ablation procedure for recurrent atrial fibrillation $(A F)$. The white arrows highlight the course of the esophagus, which is located toward the right-sided pulmonary veins $(P V)$. The red and yellow arrows point to the ablation catheter and a ring catheter in the right superior $P V$, respectively. $L A O=$ left anterior oblique.

electrograms. ${ }^{7}$ Seven patients (17\%) underwent a hybrid approach consisting of both circumferential PV ablation and complex, fractionated electrogram abaltion.

Radiofrequency energy was delivered using a 8-mm-tip ablation catheter (Navistar, Biosense Webster) with temperature and power settings of $50-55^{\circ} \mathrm{C}$ and $50-60 \mathrm{~W}$, respectively. The endpoint was reduction of the amplitude of the local electrogram by $>80 \%$ or to less than $0.1 \mathrm{mV}$. In patients who underwent electrogram-guided ablation, radiofrequency energy was delivered until voltage abatement as above, or organization of the local electrogram. Procedural endpoints also included conversion of AF to sinus rhythm, or elimination of all sites with complex electrograms within the LA.

Rhythm-control medications were prescribed for 6-12 weeks in patients with persistent AF. Rate-control medications were prescribed for patients with paroxysmal AF. Patients were monitored overnight and were discharged on subcutaneous low-molecular-weight heparin for 5 days and warfarin. Patients were seen in follow-up at 3 and 6 months, and as clinically indicated thereafter.

\section{Mapping and Ablation of Atypical Atrial Flutter}

The PV ostia were identified as described above. Atypical atrial flutter was mapped with activation mapping using an electroanatomic mapping system (CARTO; BiosenseWebster) and entrainment mapping. Radiofrequency energy was delivered at sites within the reentry circuit and extended to anatomic barriers or areas with conduction block. Catheter ablation was performed using an 8-mm-tip catheter (Navi- star, Biosense-Webster) with the same temperature and power settings as for AF, or an 3.5-mm irrigated-tip catheter (Thermocool, Biosense-Webster) with a power setting of 25-35 W and a temperature cut-off of $45^{\circ} \mathrm{C}$. The postprocedure care and follow-up were the same as after ablation of AF except that rhythm-control medications were only prescribed if the procedure was unsuccessful.

\section{Imaging of the Esophagus}

After creation of the electroanatomical map of the LA, a barium swallow was performed prior to conscious sedation. Patients were asked to swallow $5 \mathrm{cc}$ of barium paste (E-ZPaste, E-Z-EM Canada Inc., Westbury, NY, USA). Cinefluoroscopic images were recorded during the barium swallow in an anteroposterior and left anterior oblique $\left(45^{\circ}\right)$ projections (Fig. 1). An esophagram was recorded only after the patient had completely swallowed the bolus of barium, because deglutition itself may alter esophageal dimensions. The ablation catheter was then moved to the portion of the LA that was overlying the esophagus as visualized on the esophagram. The alignment of the ablation catheter with the esophagus was then confirmed in multiple fluoroscopic views. After catheter stability was confirmed for 5 seconds, the course of the esophagus was then "tagged" on the electroanatomic map. These steps were performed at three levels to determine the esophageal course with respect to the superior, mid, and inferior LA (Fig. 2). General anesthesia was not used in any of the patients. There were no instances of barium aspiration.
A

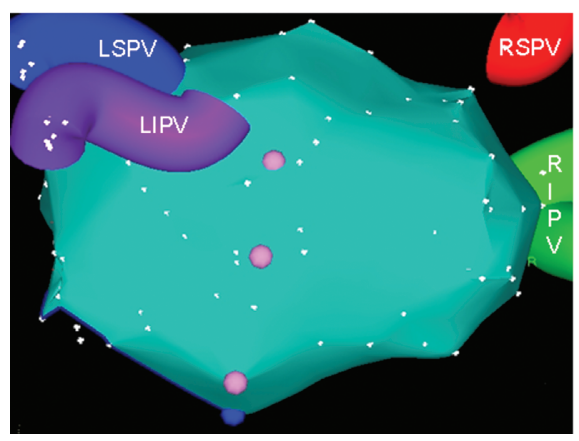

B

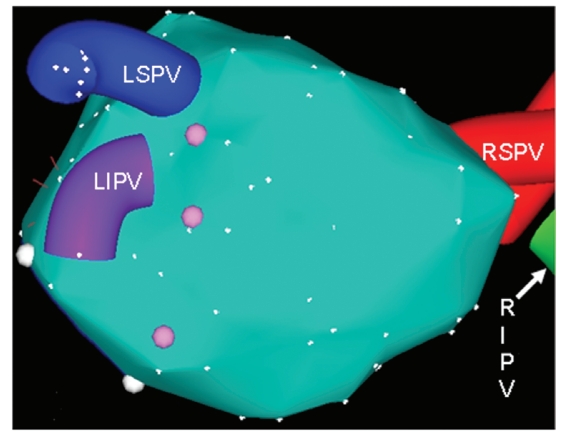

Figure 2. A: An electroanatomical map in a $P A$ view in a patient undergoing catheter ablation of AF. The esophagus (pink tags) lies near the ostia of the left-sided pulmonary veins. B: An electroanatomical map from the same patient as in panel A showing that the course of the esophagus is preserved during a repeat procedure 6 months later. $L S=$ left superior; $L I=$ left inferior $; R S=$ right superior $; R I=$ right inferior. 
A

Figure 3. Panels $A$ and $B$ show that the course of the esophagus at the first $(A)$ and second $(B)$ ablation procedures is the same, adjacent to the ostia of the right-sided PVs. The two ablation procedures were separated by 9 months.

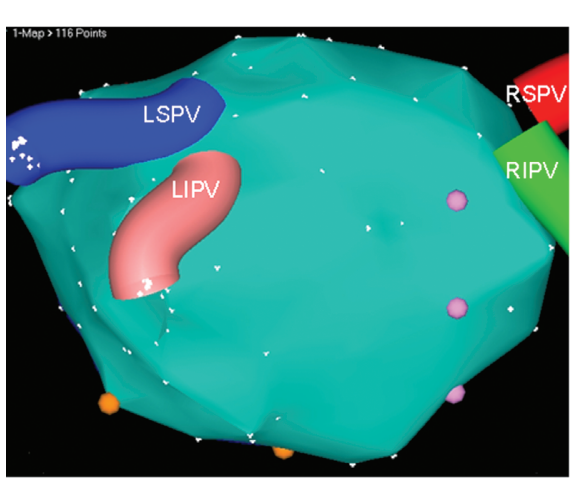

B

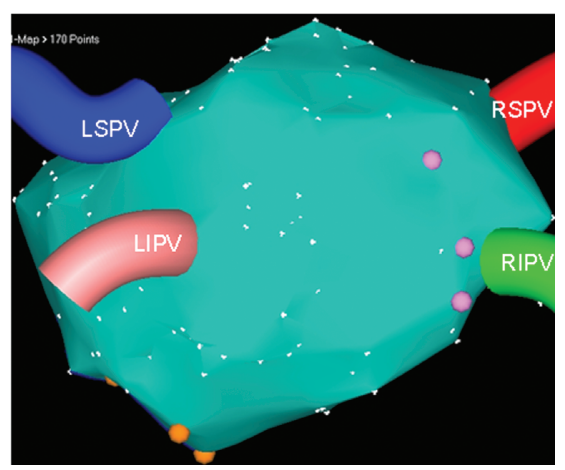

\section{Analysis of the Position of the Esophagus}

The electroanatomical maps from the 1st and 2nd ablation procedures were compared off-line by one investigator (R.K.). To minimize bias, the two 3D maps per patient were not analyzed sequentially. The position of the esophagus was classified as being adjacent to the left- or right-sided PVs or along the middle portion of the posterior LA. The distance between the esophagus and the ostia of the PVs was measured on the electroanatomical maps. Specifically, a vertical line was drawn connecting the ostial points of the ipsilateral PVs. Then, the distance from the middle of this line to the corresponding esophageal point was determined. A shift in location of the esophagus was considered to be significant if the esophagus at the time of the second ablation procedure was positioned $\geq 1.0 \mathrm{~cm}$ away from its location during the first ablation procedure. LA volume was also determined from the maps obtained from both the first and second ablation procedures using an automated function (CARTO, Biosense-Webster).

\section{Statistical Analysis}

Continuous variables are expressed as mean \pm one standard deviation and were compared using a paired $t$-test. Categorical variables were compared by a two-sided Fisher exact test. A P-value $<0.05$ indicated statistical significance.

\section{Results}

Eighty-four electroanatomical maps from 42 patients were analyzed in order to compare the position of the esophagus. The LA volumes determined from the first and second electroanatomical maps were $134 \pm 41 \mathrm{~mL}$ and $118 \pm 35 \mathrm{~mL}$, respectively $(\mathrm{P}=0.001)$. At least a $10 \%$ reduction in LA volume was noted in 24 of the 42 patients (57\%). During the first ablation procedure, the esophagus was located near the left PVs in 20 patients (48\%) (Fig. 2), near the right PVs in 13 patients (31\%) (Fig. 3), and along the mid-portion of the posterior LA in 9 patients (21\%). During the second ablation procedure, the esophagus was located near the left PVs in 22 patients (52\%), near the right PVs in 11 patients (26\%), and along the mid-portion of the posterior LA in nine patients (21\%). Quantitatively, the esophagus was positioned a mean of $23.8 \pm 13.5 \mathrm{~mm}$ from the ostia of the left PVs during the first procedure, compared to a mean of $24.6 \pm 15.9$ $\mathrm{mm}$ during the second procedure $(\mathrm{P}=0.7)$. The esophagus was positioned a mean of $41.6 \pm 13.8 \mathrm{~mm}$ from the ostia of the right PVs during the first procedure, compared to a mean of $41.7 \pm 14.0 \mathrm{~mm}$ during the second procedure $(\mathrm{P}=1.0)$ (Table 1).

In 35 of 42 patients (83\%), there was no shift in the position of the esophagus. In the other seven patients (17\%), the esophagus shifted by at least $\geq 1 \mathrm{~cm}$ (range $1.0-4.0 \mathrm{~cm}$ ) (Figs. 4 and 5). Specifically, the esophagus shifted to the leftand right-sided PVs in five and two patients, respectively.

A transesophageal echocardiogram was performed before the second ablation procedure in 29 of 42 patients (69\%). A shift in the position of the esophagus was not related to whether or not a transesophageal echocardiogram had been performed $(P=0.7)$. Further, a shift in esophageal location was not related to a reduction in LA volume $(\mathrm{P}=0.7)$.

\section{Discussion}

\section{Main Findings}

The main finding of this study is that the relationship of the esophagus with respect to the LA is unchanged during two ablation procedures separated by a mean of 7 months in $>80 \%$ of patients. Therefore, although the esophagus may be mobile during the course of an $\mathrm{AF}$ ablation procedure, ${ }^{4}$ it appears to have a resting position in the posterior mediastinum that typically remains constant. These findings demonstrate that if the position of the esophagus limits the safe delivery of radiofrequency energy during an AF ablation procedure, a second ablation procedure at a later time is likely to be met with the same limitation.

\section{Esophageal Mobility}

At first, it may seem difficult to reconcile the fact that the esophagus is "acutely" mobile during an ablation procedure, and also that its location is preserved in patients presenting for a repeat ablation procedure several months later. The results of the current study suggest that the esophagus has a

\section{TABLE 1}

Esophageal Location with Respect to the Pulmonary Veins at the First and Second Ablation Procedure

\begin{tabular}{lccc}
\hline & 1st (mm) & 2nd (mm) & P-value \\
\hline Eso-LPV & $23.8 \pm 13.5$ & $24.6 \pm 15.9$ & 0.7 \\
Eso-RPV & $41.6 \pm 13.8$ & $41.7 \pm 14.0$ & 1.0 \\
\hline
\end{tabular}

"Eso-LPV" and "Eso-RPV" refer to the distance between the esophagus and left- and right-sided pulmonary veins, respectively; "1st" and "2nd" refer to the first and second ablation procedures. 
A

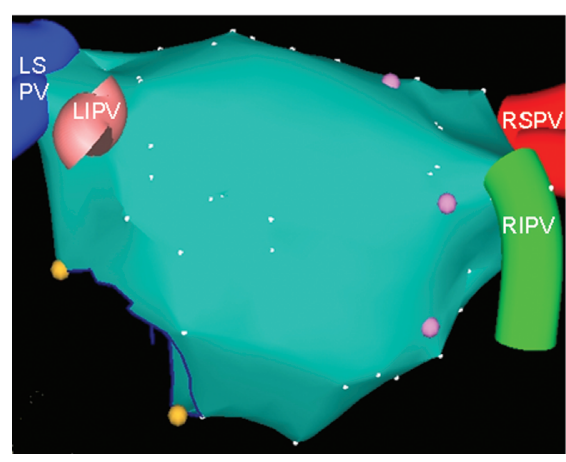

B

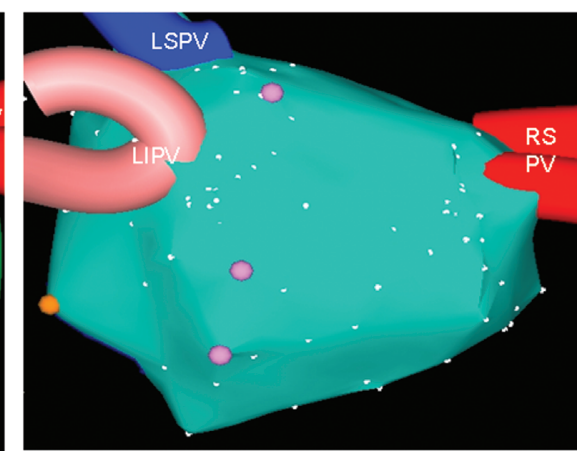

Figure 4. An example of esophageal shift. A: The esophagus was coursing near the ostia of the right-sided $P V$ s during the initial ablation procedure. $B$ : During the repeat ablation procedure 7 months later, the esophagus was near the left-sided $P V s$, representing a shift of $4 \mathrm{~cm}$. relatively stable course in the posterior mediastinum, which seems to be perturbed during the ablation procedure. This perturbation is probably imparted by peristalsis as suggested in Figure 5. Peristalsis may have been triggered by deglutition of secretions and/or barium.

It may be hypothesized that the patients in whom the position of the esophagus was stable over time are also the ones in whom it does not shift acutely during the ablation procedure. If this hypothesis were correct, esophageal stability over many months should have only been documented in one-third of the patients, because the esophagus was shown to have shifted acutely in two-thirds of the patients in a prior study. ${ }^{4}$ In contrast, the location of the esophagus was found to be stable in $>80 \%$ of the patients in the current study.

\section{Left Atrial Volume}

The LA volume at the repeat ablation procedure was $12 \%$ smaller than at the initial procedure. One possible explanation for a decrease in volume is a reverse remodeling due to catheter ablation. ${ }^{8}$ However, the fact that most of the patients undergoing a repeat procedure presented in persistent atrial fibrillation or flutter strongly argues against this hypothesis. It is possible that the decrease in volume is due to LA scarring and contraction due to multiple linear lesions.

\section{Unfavorable LA/Esophageal Topography}

Patients in whom the ablation procedure cannot be completed as planned because of the proximity of the esophagus to a critical ablation site may be more likely to experience arrhythmia recurrence and require a repeat ablation procedure. Because the same limitation is likely to be present during the second ablation procedure, it may be appropriate to consider a different ablation technique, such as cryoablation. Cryoablation may be safer than radiofrequency energy because it tends to preserve tissue architecture. However, experimental studies in animal models have arrived at conflicting conclusions regarding the safety of cryoablation near the esophagus. ${ }^{9,10}$ Our current practice in these patients is to mechanically displace the esophagus by inserting a rigid probe, as has been reported previously. ${ }^{11}$ This allows safe radiofrequency ablation without endangering the esophagus.
A

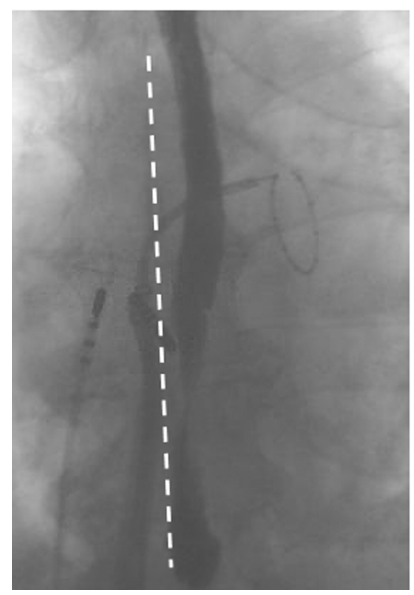

B

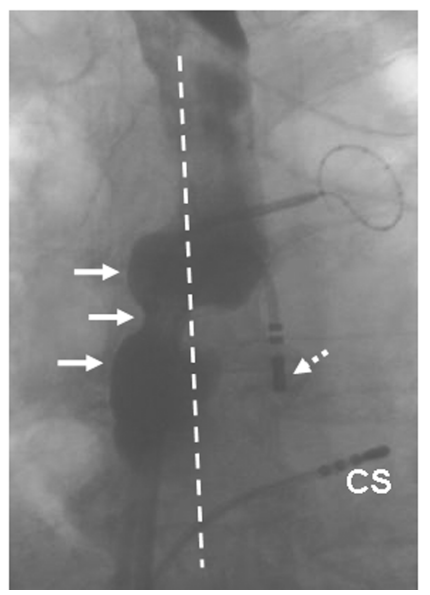

C

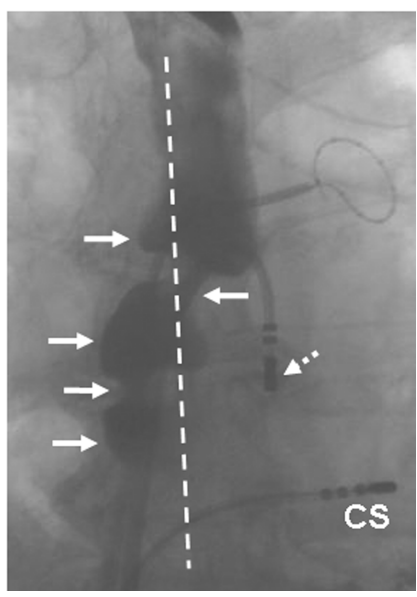

Figure 5. Esophageal mobility due to peristalsis. A: A barium swallow in an AP projection shows that the esophagus lies along the mid-portion of the posterior LA. Also seen are the ablation catheter, a catheter in the right atrium, and a ring catheter placed near the ostium of the left superior PV. The dashed line shows the extent of the spinous processes as a reference. B: Shortly after commencing ablation, esophageal peristalsis was observed (solid arrows) and the esophagus shifted to the right of the spinous processes (dashed line), posterior to the right-sided PV ostia. The dashed arrow indicates the ablation catheter. $C$ : Additional peristaltic movement of the esophagus. Also note the marked increase in the luminal diameter of the proximal esophagus. The fluoroscopic projection is the same in all panels. $C S=$ coronary sinus. 


\section{Prior Studies}

There are no prior studies concerning the temporal stability of the esophagus in patients undergoing LA ablation. A previous study examined the relationship of the LA and esophagus on computed tomography. ${ }^{12}$ However, whether the anatomic relationship of the two structures is constant over time was not explored.

\section{Limitations}

It is plausible that deglutition of barium or secretions may have perturbed the intrinsic position of the esophagus by induction of peristalsis. The smaller LA volume at the repeat ablation procedure potentially may have made it difficult to compare the location of the esophagus relative to the LA. However, the distance between the esophagus and the PVs, which generally was in the range of $2-4 \mathrm{~cm}$, is unlikely to have been affected by a small change in chamber volume.

\section{Clinical Implications}

The main clinical implication of the study is that if the initial ablation procedure was hindered by the location of the esophagus, the same limitation is likely to be present at the repeat session. However, in nearly $20 \%$ of patients the esophageal location is at least $1 \mathrm{~cm}$ removed from the original position, making reevaluation of the esophagus necessary during a repeat ablation procedure.

\section{References}

1. Pappone C, Oral H, Santinelli V, Vicedomini G, Lang CC, Manguso F, Torracca L, Benussi S, Alfieri O, Hong R, Lau W, Hirata K, Shikuma N, Hall B, Morady F: Atrio-esophageal fistula as a complication of percutaneous transcatheter ablation of atrial fibrillation. Circulation 2004;109:2724-2726.

2. Scanavacca MI, D'Avila A, Parga J, Sosa E: Left atrial-esophageal fistula following radiofrequency catheter ablation of atrial fibrillation. J Cardiovasc Electrophysiol 2004;15:960-962.
3. Oral H, Chugh A, Good E, Sankaran S, Reich SS, Igic P, Elmouchi D, Tschopp D, Crawford T, Dey S, Wimmer A, Lemola K, Jongnarangsin K, Bogun F, Pelosi F Jr, Morady F: A tailored approach to catheter ablation of paroxysmal atrial fibrillation. Circulation 2006;113:18241831.

4. Good E, Oral H, Lemola K, Han J, Tamirisa K, Igic P, Elmouchi D, Tschopp D, Reich S, Chugh A, Bogun F, Pelosi F Jr, Morady F: Movement of the esophagus during left atrial catheter ablation for atrial fibrillation. J Am Coll Cardiol 2005;46:2107-2110.

5. Cheung P, Hall B, Chugh A, Good E, Lemola K, Han J, Tamirisa K, Pelosi F Jr, Morady F, Oral H: Detection of inadvertent catheter movement into a pulmonary vein during radiofrequency catheter ablation by real-time impedance monitoring. J Cardiovasc Electrophysiol 2004;15:674-678.

6. Oral H, Scharf C, Chugh A, Hall B, Cheung P, Good E, Veerareddy S, Pelosi F Jr, Morady F: Catheter ablation for paroxysmal atrial fibrillation: Segmental pulmonary vein ostial ablation versus left atrial ablation. Circulation 2003;108:2355-2360.

7. Nademanee K, McKenzie J, Kosar E, Schwab M, Sunsaneewitayakul B, Vasavakul T, Khunnawat C, Ngarmukos T: A new approach for catheter ablation of atrial fibrillation: Mapping of the electrophysiologic substrate. J Am Coll Cardiol 2004;43:2044-2053.

8. Oral H, Pappone C, Chugh A, Good E, Bogun F, Pelosi F Jr, Bates ER, Lehmann MH, Vicedomini G, Augello G, Agricola E, Sala S, Santinelli V, Morady F: Circumferential pulmonary-vein ablation for chronic atrial fibrillation. N Engl J Med 2006;354:934 941.

9. Aupperle H, Doll N, Walther T, Kornherr P, Ullmann C, Schoon HA, Mohr FW: Ablation of atrial fibrillation and esophageal injury: Effects of energy source and ablation technique. J Thorac Cardiovasc Surg 2005;130:1549-1554

10. Ripley KL, Gage AA, Olsen DB, Van Vleet JF, Lau CP, Tse HF: Time course of esophageal lesions after catheter ablation with cryothermal and radiofrequency ablation: Implication for atrio-esophageal fistula formation after catheter ablation for atrial fibrillation. J Cardiovasc Electrophysiol 2007;18:642-646.

11. Herweg B, Johnson N, Postler G, Curtis AB, Barold SS, Ilercil A: Mechanical esophageal deflection during ablation of atrial fibrillation. Pacing Clin Electrophysiol 2006;29:957-961.

12. Lemola K, Sneider M, Desjardins B, Case I, Han J, Good E, Tamirisa K, Tsemo A, Chugh A, Bogun F, Pelosi F Jr, Kazerooni E, Morady $\mathrm{F}$, Oral H: Computed tomographic analysis of the anatomy of the left atrium and the esophagus: Implications for left atrial catheter ablation. Circulation 2004;110:3655-3660. 